\title{
Type II superlattices for infrared detectors and devices
}

\author{
D H Chow $\dagger$, R H Miles $\dagger$, J N Schulman $\nmid$, D A Collins $\ddagger$ and \\ T C McGill;
}

† Hughes Research Laboratories, Malibu, CA 90265, USA

¥California Institute of Technology, Pasadena, CA 91125, USA

\begin{abstract}
Superlattices consisting of combinations of III-V semiconductors with type II band alignments are of interest for infrared applications because their energy gaps can be made smaller than those of any 'natural' III-V compounds. Specificaliy, it has been demonstrated that both $\ln S b / \ln A s_{x} S_{1} b_{1-x}$ superlattices and $\mathrm{Ga}_{1-x} \mathrm{In}_{x} \mathrm{Sb} / \mathrm{In} A \mathrm{~s}$ superlattices can possess energy gaps in the 8-14 $\mu \mathrm{m}$ range. Our efforts have focused on the $\mathrm{Ga}_{1-x} \ln \mathrm{n}_{x} \mathrm{Sb} / \mathrm{In} A$ s system because of its extreme broken gap band alignment, which results in narrow energy gaps for very short superlattice periods. We have previously demonstrated that $\mathrm{Ga}_{0.75} \operatorname{In}_{0.25} \mathrm{Sb} / \ln \mathrm{As}$ superlattices (with periods less than $75 \AA$ ) grown on thick, stress-relaxed GaSb buffer layers on GaAs or InP substrates possess photoconductive thresholds throughout the 8-14 $\mu \mathrm{m}$ range and beyond; these superlattices simultaneously display optical absorption coefficients comparable to those of bulk $\mathrm{HgCdTe}$. We report here the use of in situ chemical doping of $\mathrm{Ga}_{1_{-}} \ln _{x} \mathrm{Sb} / \mathrm{InAs}$ superlattices to fabricate $p-n$ photodiodes. These diodes display a clear photovoltaic response with a threshold near $12 \mu \mathrm{m}$. We have also attained outstanding structural quality in $\mathrm{Ga}_{1-x} \ln \mathrm{Sb} / \ln A s$ superlattices grown on radiatively heated GaSb substrates. Cross-sectional transmission electron microscope images of these superlattices display no dislocations, while high resolution $x$-ray diffraction scans reveal sharp high-order superlattice satellites and strong Pendellösung fringes. We anticipate that this high level of structural quality will be of importance in determining material characteristics, such as carrier lifetimes, which are crucial for detector performance.
\end{abstract}

\section{Introduction}

It has been several years since the first proposal that superlattices could be used as alternative materials for infrared detection $[1,2]$. It is anticipated that this class of materials will have several advantages over bulk $\mathrm{Hg}_{1-x} \mathrm{Cd}_{x} \mathrm{Te}$ (the current industry standard) for this application:

(i) a higher degree of uniformity, which is of importance for detector arrays;

(ii) smaller leakage currents due to the suppression of tunnelling (larger effective masses) available in superlattices;

(iii) lower Auger recombination rates (in some superlattices) due to substantial splitting of the light- and heavy-hole bands and increased electron effective masses (D L Smith, private communication); and

(iv) well understood device fabrication and materials processing techniques.

Unfortunately, early attempts to realize superlattices with properties suitable for infrared detection were un- successful, largely because of the difficulties associated with epitaxial deposition of $\mathrm{HgTe} / \mathrm{CdTe}$ superlattices. More recently, significant interest has been shown in doped multi-quantum well (MQW) approaches to infrared detection, most notably $\mathrm{GaAs} / \mathrm{Al}_{x} \mathrm{Ga}_{1-x}$ As MQWs [3, 4]. However, these detectors are extrinsic in nature, and have been predicted to be limited to performance inferior to that of intrinsic detectors, including both bulk $\mathrm{HgCdTe}$ and superlattices with infrared energy gaps [5].

Superlattices composed of III-V semiconductors with type II band alignments are of interest for infrared applications because they provide the possibility of realizing materials with energy gaps smaller than those of any 'natural' III-V semiconductors. Both $\mathrm{Ga}_{1-x} \mathrm{In}_{x} \mathrm{Sb} / \mathrm{InAs}$ and $\mathrm{InSb} / \mathrm{InAs}_{x} \mathrm{Sb}_{1-x}$ superlattices have recently been demonstrated to possess energy gaps in the $8-14 \mu \mathrm{m}$ range [6-9]. Both types of superlattice rely upon an intrinsic valence to conduction band absorption process. Our efforts have focused on the $\mathrm{Ga}_{1-x} \mathrm{In}_{x} \mathrm{Sb} / \mathrm{InAs}$ materials system because of its extreme broken gap band alignment which allows the realization of $8-14 \mu \mathrm{m}$ energy gaps with very thin super- 
lattice layers $(<40 \AA)[6,10]$. The GaSb valence band edge lies approximately $100 \mathrm{meV}$ above the InAs conduction band edge at low temperature. This band alignment becomes more extreme with increasing In content in the $\mathrm{Ga}_{2-x} \mathrm{In}_{x} \mathrm{Sb}$ layers due to strain [10]. In contrast, $\mathrm{InSb} / \mathrm{InAs}_{x} \mathrm{Sb}_{1-x}$ superlattices require fairly thick layers (75 §̊) for long-wavelength infrared response. Furthermore, a lattice-matched substrate, GaSb, exists for $\mathrm{Ga}_{1-x} \mathrm{In}_{x} \mathrm{Sb} / \mathrm{InAs}$ superlattices (for specific choices of composition and layer thickness), while $\operatorname{InSb} / \operatorname{InAs}_{x} \mathrm{Sb}_{1-x}$ superlattices must be grown on relaxed buffer layers on $\mathrm{InSb}$ substrates.

We have previously demonstrated that $\mathrm{Ga}_{1-x} \mathrm{In}_{x} \mathrm{Sb} / \mathrm{InAs}$ superlattices grown on stress-relaxed $\mathrm{GaSb}$ buffer layers on GaAs or InP substrates possess energy gaps and absorption coefficients suitable for infrared detection in the 8-14 $\mu \mathrm{m}$ range. Figure 1 displays photoconductive spectra from five $\mathrm{Ga}_{1-x} \mathrm{In}_{x} \mathrm{Sb} / \mathrm{InAs}$ superlattices with varying $\mathrm{Ga}_{1-x} \mathrm{In}_{x} \mathrm{Sb}$ compositions and layer thicknesses. The spectra demonstrate that an increase in cut-off wavelength is observed when either the In content of the $\mathrm{Ga}_{1-x} \mathrm{In}_{x} \mathrm{Sb}$ layers or the InAs layer thickness is increased. Figure 2 contains a plot of the absorption coefficient measured from a $25 \AA / 41 \AA$ $\mathrm{Ga}_{0.75} \mathrm{In}_{0.25} \mathrm{Sb} / \mathrm{InAs}$ superlattice as a function of wavelength. The measured absorption is in excellent agreement with a calculated absorption coefficient which is also plotted. The calculation was performed via a twoband tight-binding model which includes the effects of strain.

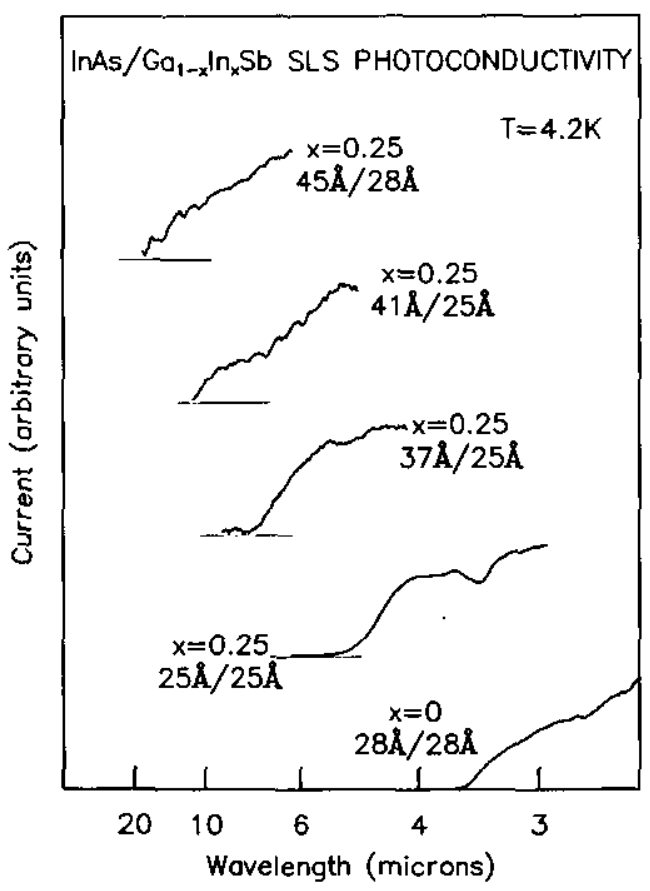

Figure 1. Photoconductivity spectra from five $\mathrm{Ga}_{1-x} \ln \mathrm{Sb} / \ln \mathrm{As}$ superlattices at $5 \mathrm{~K}$. Nominal layer thicknesses and compositions are shown. The cut-off wavelength is observed to increase with increasing in content of the $\mathrm{Ga}_{1-x} \ln _{x} \mathrm{Sb}$ layers and increasing InAs layer thickness.

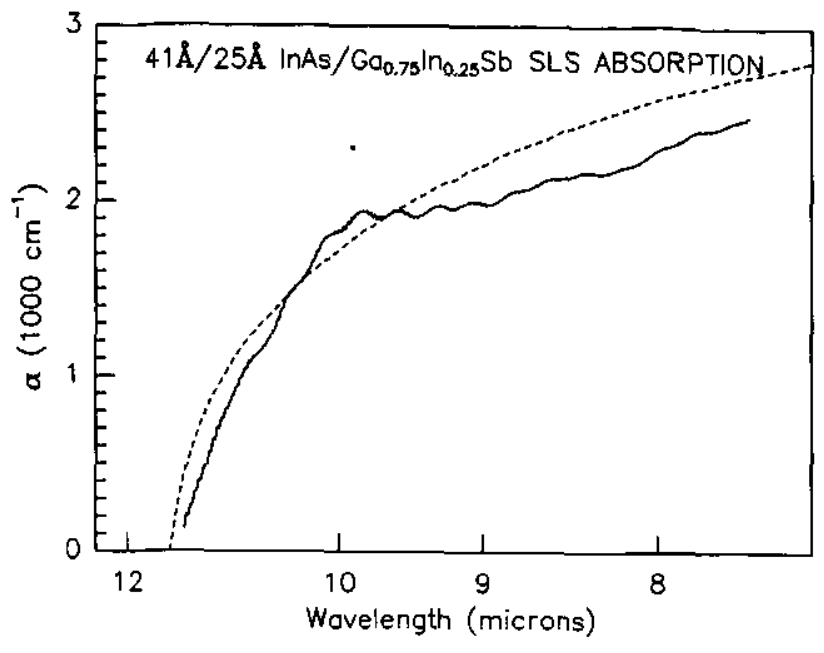

Figure 2. Measured (full curves) and calculated (broken curves) absorption coefficients for a $25 \AA / 41 \AA$

$\mathrm{Ga}_{0.75} / \mathrm{n}_{0.25} \mathrm{Sb} / \mathrm{ln} A$ s superiattice at $5 \mathrm{~K}$. The observed absorption is comparable in magnitude to that of bulk $\mathrm{Hg}_{1-x} \mathrm{Cd}_{x} \mathrm{Te}$ (with the same energy gap).

We report here the results of two more recent experiments: growth and structural characterization of $\mathrm{Ga}_{1-x} \mathrm{In}_{x} \mathrm{Sb} / \mathrm{InAs}$ superlattices grown on GaSb substrates, and fabrication of $\mathrm{p}-\mathbf{n}$ junctions in $\mathrm{Ga}_{1-x} \mathrm{In}_{x} \mathrm{Sb} / \mathrm{InAs}$ superlattices via in situ chemical doping during growth by molecular beam epitaxy (MBE). Section 2 of the paper describes the experimental procedure used to grow the samples for this study. Section 3 contains structural characterizaton of superlattices grown on $\mathrm{GaSb}$ substrates by high-resolution $\mathrm{x}$-ray diffraction (HRXRD). Section 4 discusses some considerations for doping $\mathrm{Ga}_{1_{-}} \mathrm{In}_{x} \mathrm{Sb} / \mathrm{InAs}$ superlatices to fabricate $p-n$ junctions. Photovoltaic characterization of a junction is presented. Finally, section 5 contains a summary of the results presented in the paper.

\section{Growth of $\mathrm{Ga}_{1-x} \ln _{x} \mathrm{Sb} / \ln A$ s superlattices}

All of the samples for this study were grown by MBE in a Perkin Elmer 430P system equipped with cracked arsenic and antimony sources. $\mathrm{Ga}_{1-x} \operatorname{In}_{x} \mathrm{Sb} / \mathrm{InAs}$ superlattices were deposited on three different types of substrate: $\mathrm{GaAs}$, InP and $\mathrm{GaSb}$. In the cases of lattice-mismatched GaAs and InP substrates, thick $(0.5-1.0 \mu \mathrm{m})$ stress-relaxed GaSb buffer layers were deposited prior to growth of the superlattices. The procedure used for deposition of these buffer layers has been described elsewhere $[11,12]$. Superlattices grown in this manner yield excellent surface morphology and sharp $\Theta / 2 \Theta$ x-ray diffraction satellites. However, cross sectional transmission electron microscope (TEM) images reveal dislocation densities as high as $10^{9} \mathrm{~cm}^{-2}$ [12] in these samples; high-resolution x-ray diffraction satellites from these samples are characteristically broad and weak. The observed threading dislocations appear to result solely from relaxation of the $\mathrm{GaSb}$ buffer layers, with no evidence of relaxation observed in the individual superlattice layers nor be- 
tween the superlattices and buffer layers. For growth of $\mathrm{Ga}_{1-x} \mathrm{In}_{x} \mathrm{Sb} / \mathrm{InAs}$ superlattices on $\mathrm{GaSb}$ substrates, round (30 $\mathrm{mm}$ diameter), (100)-oriented wafers were used. These substrates were radiatively heated (Infree) for MBE growth via custom-designed molybdenum wafer holders. The use of solderless substrate mounting was found to be of importance in preventing warping and/or cracking of the substrate due to thermal stress. The substrates were chemically cleaned prior to growth by a standard degrease and $10 \mathrm{~min}$ etch in $\mathrm{Br}_{2}: \mathrm{HNO}_{3}: \mathrm{HCl}: \mathrm{CH}_{3} \mathrm{COOH}(0.35: 2: 36: 460)$ (S J Eglash, private communication). In situ cleaning consisted of outgassing at $250^{\circ} \mathrm{C}$ in an ultrahigh vacuum buffer chamber, followed by heating in an $\mathrm{Sb}$ flux to the oxide desorption temperature $\left(\approx 500^{\circ} \mathrm{C}\right)$ in the growth chamber. Reflection high-energy electron diffraction (RHEED) was used to monitor the oxide desorption process.

Typical superlattice layer thicknesses and compositions are eight monolayers $(25 \AA)$ of $\mathrm{Ga}_{0.73} \operatorname{In}_{0.27} \mathrm{Sb}$ and thirteen monolayers $(39 \AA)$ of InAs. These parameters have previously been shown to result in a superlattice with an energy gap near $100 \mathrm{meV}(12 \mu \mathrm{m})$ [6]. Total superlattice thicknesses range from 40 to 80 periods $(0.25$ to $0.5 \mu \mathrm{m}$ ). At each interface of the superlattice, growth was interrupted (no group III flux) in an $\mathrm{Sb}_{2}$ flux for $5 \mathrm{~s}$.

During growth of the $\mathrm{Ga}_{1-x} \operatorname{In}_{x} \mathrm{Sb}$ layers, a $1 \times 3$ RHEED pattern is observed, while InAs layers display a $1 \times 2$ pattern. As the substrate temperature is varied, a transition from $1 \times 3$ to $1 \times 5$ in the RHEED pattern from the $\mathrm{Ga}_{0.73} \mathrm{In}_{0.27} \mathrm{Sb}$ surface during a growth interruption (in an $\mathrm{Sb}_{2}$ flux) is observed. The transition point is approximately $380^{\circ} \mathrm{C}$. The superlattices studied here were grown at substrate temperatures just below this point. A similar transition in surface reconstruction also occurs at $\approx 440^{\circ} \mathrm{C}$ for a GaSb surface in an $\mathrm{Sb}_{2}$ flux, and at $\approx 410^{\circ} \mathrm{C}$ during growth of GaSb. These observations suggest that the $1 \times 3$ to $1 \times 5$ transition in antimonide surface reconstruction is caused by an increase in $\mathrm{Sb}$ surface coverage at temperatures below the transition temperature. This explanation is supported by the fact that incorporation of As in antimonide layers has been observed to increase with substrate temperature [11], and is nearly eliminated in antimonide layers grown below the transition temperature.

\section{Structural characterization}

We anticipate that the structural quality of $\mathrm{Ga}_{1-x} \operatorname{In}_{x} \mathrm{Sb} / \mathrm{InAs}$ superlattices will have profound effects upon material properties, such as carrier lifetimes and mobilities, which are crucial for detector performance. Although some workers have reported success in reducing dislocation densities in relaxed buffer layers [13], high structural quality is commonly obtained only on lattice-matched substrates.

Figure 3(a) displays high-resolution $\mathrm{x}$-ray diffraction scans (rocking curves) from $\mathrm{Ga}_{1-x} \mathrm{In}_{x} \mathrm{Sb} / \mathrm{InAs}$ superlattices grown on InP (broken curve) and $\mathrm{GaSb}$ (full curve)
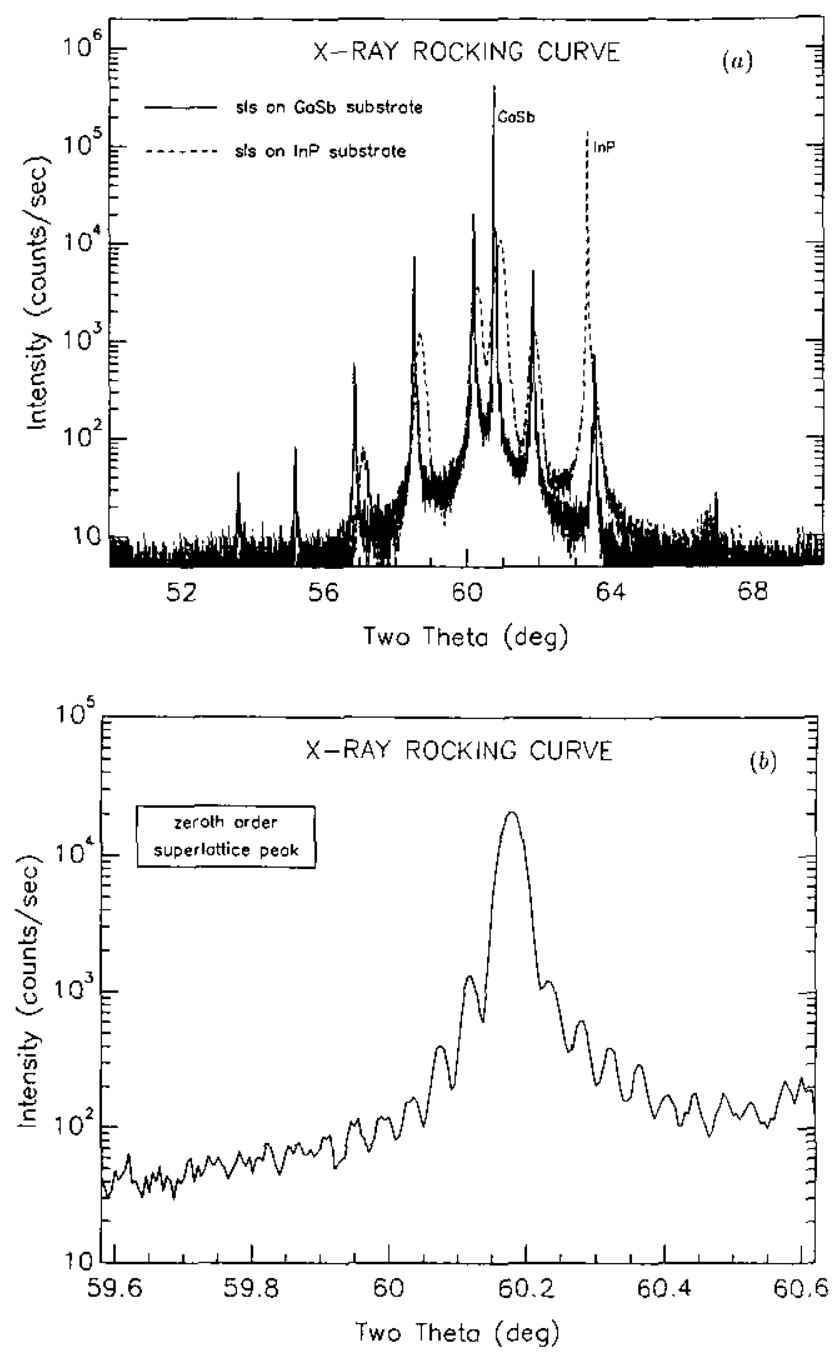

Figure 3. (a) High-resolution x-ray diffraction from $\mathrm{Ga}_{1-x} \ln \mathrm{n}_{x} \mathrm{Sb} / \mathrm{InAs}$ superlattices (sls) grown on InP (broken curve) and GaSb (full curve) substrates. The superlattices were grown with similar layer thicknesses and composition, under the growth conditions described in section 2. A peak due to the GaSb buffer layer grown on the $\ln P$ substrate prior to deposition of the superlattice is also visible in the broken curve.

(b) Rocking-curve data from the superlattice grown on GaSb over a small angular range. Clear Pendellösung fringes are observed.

substrates. The diffraction data were taken with a $\mathrm{Cu} \mathrm{K} \alpha$ source passed through a four-crystal Ge monochromator. Long counting times (15 s) were used to improve signal-to-noise ratio in the scans. The two superlattices were both grown with a nominal In content of $x=0.27$ in the $\mathrm{Ga}_{1-x} \operatorname{In}_{x} \mathrm{Sb}$ layers, with growth conditions as described in section 2. The period of the superlattice grown on $\mathrm{GaSb}$ is slightly shorter $(61 \AA)$ than that of the superlattice grown on InP ( $64 \AA)$, as evidenced by its slightly greater $\mathrm{x}$-ray satellite spacing. Examination of figure 3(a) reveals that $\mathrm{x}$-ray diffraction satellites from the sample grown on $\mathrm{GaSb}$ are sharper, persistent to higher order, and more intense than those from the sample grown on InP. All of these features are indications of dramatic differences in structural quality between the two samples. It should be noted that we have 
attempted several different growth schemes to reduce threading dislocation densities in $\mathrm{GaSb}$ buffer layers grown on GaAs and InP substrates, including additional strained-layer superlattices, annealing and encapsulated annealing. None of these approaches resulted in highresolution $x$-ray diffraction significantly better than that shown in the broken curve of Figure 3(a).

Figure $3(b)$ displays a plot of the diffraction data from the sample grown on GaSb over a small angular range, revealing clear Pendellösung fringes around the zerothorder superlattice satellite. Theoretical simulations confirm that the width of this peak is determined by the thickness of the superlattice $(0.25 \mu \mathrm{m})$, as opposed to irregularities in superlattice period or lattice spacing. Consistent with this finding, we were unable to detect the presence of any dislocations in cross sectional TEM images of a superlattice grown on a GaSb substrate. The ability to grow $\mathrm{Ga}_{1-x} \mathrm{In}_{x} \mathrm{Sb} / \mathrm{In} A$ s superlattices which are dislocation free may be anticipated to have positive effects upon material properties which are crucial for infrared detectors. For example, carrier diffusion lengths may be limited by scattering from threading dislocations. Hence, the availability of a lattice-matched substrate represents a significant advantage for detectors based on $\mathrm{Ga}_{1-x} \mathrm{In}_{x} \mathrm{Sb} / \mathrm{InAs}$ superlattices relative to materials which must be grown on relaxed buffer layers.

\section{4. p-n junctions}

The ability to dope an infrared material both $\mathrm{p}$ type and $\mathrm{n}$ type is necessary for the realization of photovoltaic detectors. One significant advantage which III-V semiconductors hold over II-VI compounds is the greater availability of well-behaved substitutional impurity dopants. For III-V MBE growth, silicon and beryllium are by far the most popular dopants. It has recently been demonstrated that silicon is a well-behaved p-type dopant for GaSb [14], while it is an n-type dopant for InAs. Beryllium is known to be an acceptor for all of the conventional III-V compounds. Hence, it should be possible to dope $\mathrm{Ga}_{1-x} \mathrm{In}_{x} \mathrm{Sb} / \mathrm{InAs}$ superlattices $\mathrm{p}$ type by co-deposition of Be during growth of either or both constituent layers, or by co-deposition of $\mathrm{Si}$ during growth of $\mathrm{Ga}_{1-x} \mathrm{In}_{x} \mathrm{Sb}$ layers only (assuming that the diffusion of $\mathrm{Si}$ atoms is limited). Conversely, co-deposition of Si during growth of InAs layers should result in an n-type superlattice; we have observed well-behaved ntype doping of $\mathrm{Ga}_{1-x} \operatorname{In}_{x} \mathrm{Sb} / \mathrm{InAs}$ superlattices via this in situ technique up to levels of $1 \times 10^{18} \mathrm{~cm}^{-3}$. Higher levels of n-type doping have not yet been attempted, and are probably unnecessary for photovoltaic detectors.

Although intentional doping in $\mathrm{Ga}_{1-x} \mathrm{In}_{x} \mathrm{Sb} / \mathrm{InAs}$ superlattices is fairly straightforward, achieving low background (unintentional) carrier concentrations may be more difficult. To date, we have consistently observed background p-type doping in $\mathrm{Ga}_{1-x} \operatorname{In}_{x} \mathrm{Sb} / \mathrm{InAs}$ superlattices, with carrier concentrations of approximately $5 \times 10^{16} \mathrm{~cm}^{-3}$ at low temperatures. It is highly desirable to decrease this background concentration as Auger

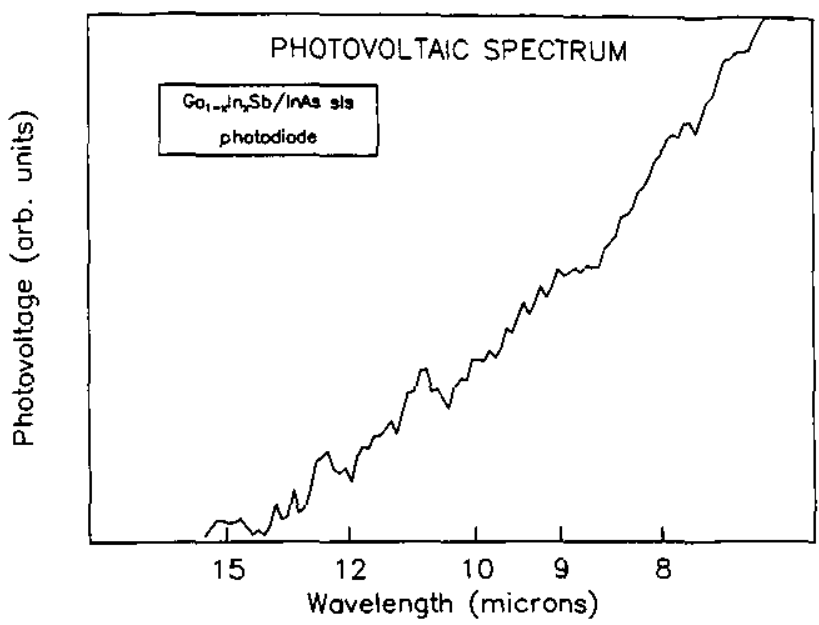

Figure 4. Photovoltaic spectrum from a $24 \AA / 40 \AA$ $\mathrm{Ga}_{0.75} \mathrm{In}_{0.25} \mathrm{Sb} / \mathrm{InAs}$ photodiode at $5 \mathrm{~K}$. The spectrum displays a threshold near $12 \mu \mathrm{m}$.

lifetimes and depletion lengths are strongly dependent upon carrier densities. Recent efforts directed towards determining the source of the background acceptors will be reported elsewhere [15].

Figure 4 displays a photovoltaic spectrum taken from a $24 \AA / 40 \AA \mathrm{Ga}_{0.75} \operatorname{In}_{0.25} \mathrm{Sb} / \mathrm{InAs}$ superlattice photodiode The p-n junction was formed by growing $0.25 \mu \mathrm{m}$ of nominally undoped superlattice material on top of $0.25 \mu \mathrm{m}$ of intentionally $\mathrm{n}$-doped material. The two sides of the junction had the same superlattice layer thicknesses and composition. Photodiodes were fabricated by chemically etching mesas [6] to isolate the junction and by depositing Al contact metal on both the tops of the mesas and the etched surface. This chip was then mounted on a header with a hole in its base for backside illumination, and wire bonds were connected to the two terminal devices. The spectrum in figure 4 was taken by using one of the photodiodes as a detector in a BOMEM Fourier transform infrared spectrometer. A clear photovoltaic response is observed from the photodiode, with a threshold near $12 \mu \mathrm{m}$, consistent with the energy gap expected from this superlattice. This demonstration of photovoltaic response verifies that photovoltaic detectors can be straightforwardly realized in the $\mathrm{Ga}_{1-x} \mathrm{In}_{x} \mathrm{Sb} / \mathrm{In} \mathrm{As}$ materials system.

\section{Summary}

Infrared detectors based on III-V compounds are highly desirable as an alternative to $\mathrm{Hg}_{1-x} \mathrm{Cd}_{x} \mathrm{Te}$, because of their materials processing and uniformity advantages. Type Il superlattices offer the possibility of creating III-V materials with cut-off wavelengths in the $8-14 \mu \mathrm{m}$ range which simultaneously possess the high detectivities and operating temperatures of an intrinsic detector. Of the two material systems which have been demonstrated to have long-wavelength infrared response, $\mathrm{Ga}_{1-x} \mathrm{In}_{x} \mathrm{Sb} / \mathrm{InAs}$ has two significant advantages over $\mathrm{InSb} / \mathrm{InAs}_{x} \mathrm{Sb}_{1-x}$ : energy gaps in the $8-14 \mu \mathrm{m}$ range are 
compatible with both reasonable interlayer strains and strong absorption coefficients, and $\mathrm{Ga}_{1-x} \mathrm{In}_{x} \mathrm{Sb} / \mathrm{InAs}$ superlattices can be grown lattice-matched to $\mathrm{GaSb}$ substrates. We have demonstrated experimentally that large absorption coefficients are compatible with longwavelength response in $\mathrm{Ga}_{1-x} \mathrm{In}_{x} \mathrm{Sb} / \mathrm{InAs}$ superlattices, and that nearly ideal structural quality can be obtained for $\mathrm{Ga}_{1-x} \mathrm{In}_{x} \mathrm{Sb} / \mathrm{InAs}$ superlattices grown on radiatively heated GaSb substrates. In addition, we have recently demonstrated photovoltaic response at $12 \mu \mathrm{m}$ from an as-grown $p-n$ junction. These results are promising for the eventual realization of material properties suitable for high-performance detectors.

\section{Acknowledgments}

The authors gratefully acknowledge helpful discussions with A T Hunter, M H Young, R Baron, T C Hassenberg, D L Smith (Los Alamos National Laboratories) and C Mailhiot (Lawrence Livermore Laboratories). The TEM results described in the paper were provided by W J Hamilton of Santa Barbara Research Center. Vital technical assistance was provided by L D Warren, K T Miller and C Hauessler. Parts of this work were performed under Contract nos N00014-89-C-0203 and N00014-89-J-3196 from the Defense Advanced Research Projects Agency and the Office of Naval Research.

\section{References}

[1] Schulman J N and McGill T C 1979 Appl. Phys. Lett. 34663

[2] Smith D L, McGill T C and Schulman J N $1983 \mathrm{Appl}$. Phys. Lett. 43180

[3] Levine B F, Bethea C G, Hasnain G, Shen V O, Pelve E, Abbott R R and Hsieh S J 1990 Appl. Phys. Lett. 56851

[4] Levine B F, Gunapala S D and Kopf R F 1991 Appl. Phys. Lett. 581551

[5] Kinch M A and Yariv A 1989 Appl. Phys. Lett. 552093

[6] Miles R H, Chow D H, Schulman J N and McGill T C 1990 Appl. Phys. Lett. 57801

[7] Campbell I H, Sela I, Laurich B K, Smith D L, Bolognesi C R, Samoska L A, Gossard A C and Kroemer H 1991 Appl. Phys. Lett. 5984

[8] Kurtz S R, Biefeld R M, Dawson L R, Fritz I J and Zipperian T E 1988 Appl. Phys. Lett. 531961

[9] Kurtz S R, Dawson L R, Biefeld R M, Fritz I J and Zipperian T E 1989 IEEE Electron Device Lett. 10 150

[10] Smith D L and Maihiot C 1987 J. Appl. Phys. 622545

[11] Chow D H, Miles R H, Söderström J R and McGill T C 1990 J. Vac. Sci. Technol. B 8710

[12] Chow D H, Miles R H, Nieh C W and McGill T C 1991 J. Cryst. Growth 11168

[13] Yamaguchi M 1991 J. Mater. Res. 6376

[14] Rossi T M, Collins D A, Chow D H and McGill T C 1990 Appl. Phys. Lett. 572256

[15] Chow D H and Miles R H unpublished 